\title{
PODA DE RAÍZES E ADUBAÇÃO PARA CRESCIMENTO DO CAFEEIRO CULTIVADO EM COLUNAS DE SOLO ${ }^{(1)}$
}

\author{
Víctor Hugo Alvarez V. ${ }^{(2)}$, Luiz Eduardo Dias ${ }^{(2)}$, Paulo Batista Leite ${ }^{(3)}$, \\ Ronessa Bartolomeu de Souza ${ }^{(4)}$ \& Emerson Silva Ribeiro Junior ${ }^{(5)}$
}

\begin{abstract}
RESUMO
Em casa de vegetação, foram estudados os efeitos de níveis de fertilidade, poda de raízes e adubação localizada sobre o crescimento da parte aérea e do sistema radicular de plantas de cafeeiro cultivar Catuaí Vermelho. Amostras subsuperficiais ( 30 a $70 \mathrm{~cm}$ ) de um LVA de Viçosa-MG foram acondicionadas em colunas de PVC compostas por três anéis de $15 \mathrm{~cm}$ de altura e $20 \mathrm{~cm}$ de diâmetro. Quatro tratamentos com três níveis de fertilidade [baixo (FB); médio (FM); alto (FA) e médio sem corte de raízes, em apenas dois anéis (FM2)], foram instalados antes do plantio, utilizando três níveis de calagem e três doses de esterco de galinha. As doses de $\mathrm{P}$ e $\mathrm{K}$ aplicadas no plantio foram inversamente proporcionais aos níveis de fertilidade propostos. Oito meses após o plantio, o anel inferior foi retirado, podando-se o sistema radicular. As amostras de solo do anel inferior foram substituídas por novas amostras nas quais foram aplicadas quatro adubações localizadas [baixa (AB); média (AM); alta (AA) e média mais esterco de galinha (AM2)]. Dezessete meses após o plantio, foram feitas avaliações da parte aérea e do sistema radicular. A altura das plantas e o número de ramos decresceram linearmente com o nível de fertilidade, em razão das menores doses de $\mathbf{P}$ e $\mathrm{K}$ adicionadas na adubação de plantio. Esta tendência também foi observada na produção de matéria seca da parte aérea; entretanto, o crescimento do sistema radicular não foi afetado. A poda de raízes não influiu no crescimento e na produção de matéria seca da parte aérea e das raízes, exceto quando se adicionou esterco de galinha na adubação localizada no nível de fertilidade média, promovendo, neste caso, efeito negativo. As adubações localizadas no nível de fertilidade baixo não afetaram o crescimento da parte aérea nem do sistema radicular; no nível médio, verificou-se efeito quadrático positivo e, no
\end{abstract}

(1) Recebido para publicação em novembro de 2003 e aprovado em setembro de 2005.

(2) Professor do Departamento de Solos, Universidade Federal de Viçosa - UFV. CEP 36570-000 Viçosa (MG). Bolsista CNPq. E-mail: vhav@ufv.br; ledias@ufv.br

(3) Engenheiro-Agrônomo, MS, Pioneer Sementes Ltda. CEP 75503-970 Itumbiara (GO). E-mail: paulobleite@terra.com

(4) Pesquisadora, DS., Embrapa Hortaliças. Rod. BR 060, km 9, Caixa Postal 218, CEP 70359-970 Brasília (DF). E-mail: ronessa@cnph.embrapa.br

${ }^{(5)}$ Engenheiro-Agrônomo, DS., COAGR-CNPq, SEPN 509, Bloco A, Ed. Nazir I, Sala 301, CEP 70750-501 Brasília (DF). Bolsista do CNPq. E-mail: eribeiro@cnpq.br 
nível alto, observou-se aumento linear no crescimento das plantas de café, mostrando que as adubações de plantio baseadas nos níveis de fertilidade foram suficientes no nível baixo, equilibradas no nível médio e insuficientes no nível alto.

Termos de indexação: Coffea arabica, corte de raízes, fertilização.

\title{
SUMMARY: ROOT PRUNING AND FERTILIZATION FOR GROWTH OF COFFEE PLANTS CULTIVATED IN SOIL COLUMNS
}

\begin{abstract}
The effects of soil fertility levels, root pruning and localized fertilization on root and shoot growth in 'Catuaí' coffee plants were studied in a greenhouse experiment. Subsuperficial (30-70 cm) samples of a Red-Yellow Latosol (Oxisol) were packed in PVC columns consisting of three $15 \mathrm{~cm}$ high rings of $20 \mathrm{~cm}$ diameter. Four treatments with three fertility levels [low (FB), medium (FM) and high (FA), and medium without root pruning with only two rings (FM2)] were installed before planting by adding three levels of liming and three doses of poultry manure. The $P$ and $K$ doses at planting were applied in inverse amounts of the soil fertility levels. After eight months of cultivation, the lowest ring was removed, and roots were pruned. This ring was replaced by another one filled with soil with four fertilization rates [low ( $A B)$, medium (AM), medium plus poultry manure (AM2) and high $(A A)$ ], representing localized fertilization. After 17 months of cultivation the shoot and root system were evaluated. Plant height and number of branches decreased with the level of soil fertility, due to the lower $P$ and $K$ doses applied along the increasing fertility level. The same trend was observed for shoot dry matter production, but root system growth was not affected. Growth and dry matter production of shoots and roots was not affected by root pruning, except when poultry manure was applied in localized fertilization at medium fertility level, at which a negative effect was observed. The localized fertilization had no affect on shoot and root growth in the FB pots, but caused positive and quadratic effects in the FM pots and a linear increase in the growth of 'Catuai' coffee plants for the FA treatment. The results showed that planting fertilization as a function of the fertility level was sufficient for FB, balanced for FM and insufficient for FA.
\end{abstract}

Index Terms: Coffea arabica, root pruning, fertilization.

\section{INTRODUÇÃO}

Em decorrência do elevado grau de intemperismo, associado ao caráter ácido de grande parte dos solos sob cerrado (Ritchey et al., 1980; Demattê, 1981; Lopes, 1983), o cultivo do cafeeiro nesses solos requer adubações periódicas que, normalmente, são realizadas em cobertura.

A aplicação superficial de fertilizantes leva a indagações em torno do aproveitamento, por parte da planta, dos nutrientes adicionados, principalmente, em se tratando de adubos fosfatados, dada a baixa mobilidade do P no solo.

Uma das soluções para esse problema é a aplicação de elevadas doses de P no substrato para a formação das mudas, aumentando as reservas internas do nutriente, que poderão ser utilizadas, posteriormente, em condições de campo. No entanto, tal medida pode ser paliativa se o teor de $\mathrm{P}$ no solo em campo for baixo, pois as mudas de café parecem ser pouco eficientes na absorção de $\mathrm{P}$ por apresentarem sistema radicular ainda reduzido, ou por terem pequena capacidade de absorver este nutriente (Pozza et al., 2002).

Outra alternativa seria a localização do $\mathrm{P}$, por meio da aplicação de fosfatos solúveis em sulco ao lado das plantas, aumentando, assim, a disponibilidade do nutriente (Leite, 1991; Fonseca, 1995). Entretanto, por apresentar uma malha de raízes absorventes na superfície do solo (Silva, 1960; Suarez de Castro \& Rodriguez, 1963), a abertura do sulco pode resultar no corte de raízes do cafeeiro. A poda de raízes pode ser uma alternativa para a economia de água e maximização da absorção de nutrientes de culturas florestais em áreas sujeitas a grandes déficits hídricos (Ong et al., 2002; Woodall \& Ward, 2002). Bar-tal et al. (1995), trabalhando com produção de tomate em solução nutritiva, observaram aumentos na absorção de $\mathrm{NO}_{3}{ }^{-}$e de $\mathrm{K}$ em plantas submetidas à poda de raízes, em virtude do aumento na produção de raízes que resultam em incremento do número de raízes finas metabolicamente mais ativas. 
Da Matta et al. (1999) observaram que uma suplementação nitrogenada não preveniu o declínio de crescimento em plantas de café instaladas em campo na estação fria; no entanto, nas raízes, a adubação nitrogenada esteve associada à manutenção da atividade da enzima nitrato redutase. Desta forma, torna-se necessário avaliar a capacidade de rebrota do sistema radicular do cafeeiro e a influência da fertilidade do solo e adubação da cultura sobre o processo.

O presente trabalho objetivou avaliar o crescimento da parte aérea e do sistema radicular de mudas de cafeeiro cultivadas em colunas de solo, com diferentes níveis de fertilidade e adubações de plantio, bem como determinar o efeito da poda de raízes sobre o sistema radicular e sobre a parte aérea de mudas crescidas em solos que receberam diferentes adubações localizadas.

\section{MATERIAL E MÉTODOS}

O trabalho foi realizado em casa de vegetação, onde se instalaram colunas de PVC rígido com $50 \mathrm{~cm}$ de altura e $20 \mathrm{~cm}$ de diâmetro, nas quais foram acondicionadas subamostras de uma amostra retirada da camada de 30 a $70 \mathrm{~cm}$ de profundidade de um Latossolo Vermelho-Amarelo ácrico (LVA) de Viçosa-MG (Quadro 1). As colunas foram montadas com três anéis, sendo o inferior e o mediano de $15 \mathrm{~cm}$ cada e o anel superior de $20 \mathrm{~cm}$ de altura com os $5 \mathrm{~cm}$ superiores sem solo.

Os tratamentos corresponderam a um fatorial $(3+1)(3+1)$, sendo quatro tratamentos com três níveis de fertilidade propostos numa primeira etapa, antes do plantio, e quatro adubações localizadas com três doses, aplicadas oito meses após o plantio. As adubações localizadas foram feitas nos anéis que substituíram o anel inferior das colunas, durante a segunda etapa do experimento. Os 16 tratamentos foram distribuídos em blocos casualizados, com quatro repetições.

Na primeira etapa do experimento, antes do plantio, utilizando diferentes quantidades de esterco de galinha e uma mistura corretiva aplicadas em todo o volume de solo da coluna (Quadro 2), foram propostos quatro tratamentos com três níveis de fertilidade: fertilidade baixa (FB); fertilidade média (FM); fertilidade alta (FA) e fertilidade média sem corte de raízes em apenas dois anéis (FM2). Ressalta-se que os três primeiros tratamentos (FB, FM e FA) foram montados em três anéis sobrepostos para posterior poda de raízes, e o último, FM2, sem o anel inferior.

Para o cálculo da necessidade de calagem, utilizou-se o método-padrão recomendado para Minas Gerais, com modificações sugeridas por Freire et al. (1984) para o cultivo do cafeeiro. A mistura corretiva aplicada foi composta por $75 \%$ de calcário dolomítico e $25 \%$ de gesso, em massa.

Quadro 1. Características químicas e físicas do Latossolo Vermelho-Amarelo de Viçosa utilizado no experimento

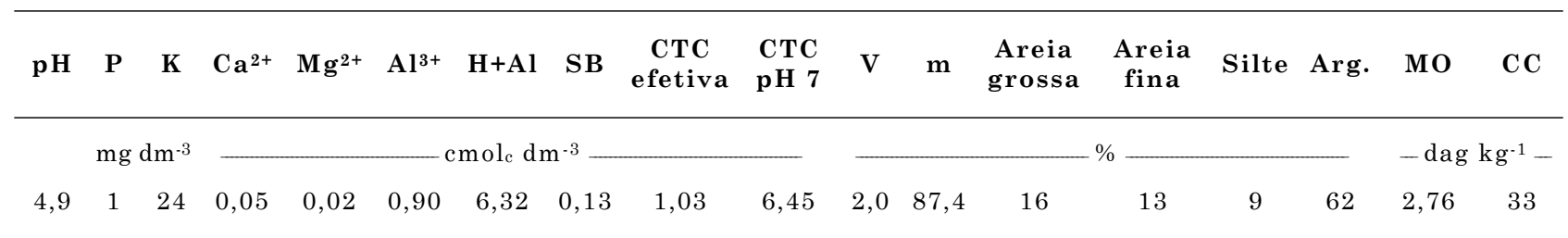

PH em $\mathrm{H}_{2} \mathrm{O}, 1: 2,5$; $\mathrm{P}$ e K disponíveis, extrator Mehlich-1 (Vettori, 1969); $\mathrm{Ca}^{2+}, \mathrm{Mg}^{2+}, \mathrm{Al}^{3+}$ trocáveis, extrator $\mathrm{KCl}_{1} \mathrm{~mol} \mathrm{~L}^{-1}(\mathrm{Vettori}$, 1969); $\mathrm{H}+\mathrm{Al}$, extrator $\mathrm{Ca}(\mathrm{OAc})_{2} 0,5 \mathrm{~mol} \mathrm{~L}^{-1}, \mathrm{pH} 7$; Areia por peneiramento; Argila, método da pipeta; MO, matéria orgânica pelo método Walkley \& Black (Jackson, 1976); CC, capacidade de campo, método da coluna transparente (Fernandes, 1967).

Quadro 2. Doses de esterco de galinha e da mistura corretiva correspondentes aos níveis de fertilidade propostos antes do plantio

\begin{tabular}{cccc}
\hline Nível de fertilidade(1) & Esterco de galinha & Mistura corretiva( \\
\hline & $\mathrm{t} \mathrm{ha}^{-1}$ & $\mathrm{NC}(3)$ & $\mathrm{t} \mathrm{ha}^{-1}$ \\
FB & 0 & 0,25 & 1,18 \\
FM & 10 & 0,75 & 3,55 \\
FM2 & 10 & 0,75 & 3,55 \\
FA & 20 & 1,25 & 5,91 \\
\hline
\end{tabular}

(1) Nível de fertilidade: $\mathrm{FB}=$ fertilidade baixa; $\mathrm{FM}=$ fertilidade média; $\mathrm{FM} 2=$ fertilidade média, sem corte de raízes e $\mathrm{FA}=$ fertilidade

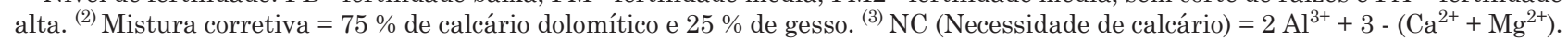


Após a incorporação do esterco e da mistura corretiva, o solo foi incubado por um período de 40 dias, para, a seguir, ser colocado nas colunas de PVC. Nessa ocasião, o solo contido no anel superior de cada coluna recebeu adubação fosfatada na forma de superfosfato triplo, em quantidade inversa aos níveis de fertilidade propostos (Quadro 3), equivalente à adubação de plantio recomendada por Freire et al. (1984) para o cultivo do cafeeiro. Em seguida, transplantou-se em cada coluna uma muda, com seis pares de folhas, mudas de cafeeiro (Coffea arábica $\mathrm{L}$ ) cultivar Catuaí Vermelho CH 2077-2-5-99 (IAC 99), provenientes do Viveiro do Fundão, do Departamento de Fitopatologia da Universidade Federal de Viçosa.

Como adubação de plantio, foram ainda aplicados $\mathrm{N}$, na forma de uréia, e K, na forma de cloreto de K (Quadro 3). Similarmente ao P, o K foi aplicado em quantidade inversa aos níveis de fertilidade propostos. As adubações de $\mathrm{N}$ e K em cobertura, aplicadas em solução, foram parceladas em seis vezes, sendo a primeira 40 dias após o plantio e as demais em intervalos de 30 dias.

Aos 120 dias do plantio, aplicou-se em cobertura uma solução que continha micronutrientes nas seguintes concentrações, em mg dm ${ }^{-3}: \mathrm{Zn}(4,00), \mathrm{Cu}$ $(1,33)$, Mo $(0,15)$, B $(0,81)$ e Mn $(3,64)$, nas formas de $\mathrm{ZnCl}_{2}$ e $\mathrm{ZnSO}_{4} \cdot 7 \mathrm{H}_{2} \mathrm{O}, \mathrm{CuCl}_{2} \cdot 2 \mathrm{H}_{2} \mathrm{O}$ e $\mathrm{CuSO}_{4} \cdot 5 \mathrm{H}_{2} \mathrm{O}$, $\mathrm{Na}_{2} \mathrm{MoO}_{4} \cdot 2 \mathrm{H}_{2} \mathrm{O}$ e $\left(\mathrm{NH}_{4}\right)_{6} \mathrm{Mo}_{7} \mathrm{O}_{24} \cdot 4 \mathrm{H}_{2} \mathrm{O}, \mathrm{H}_{3} \mathrm{BO}_{3}$ e $\mathrm{MnCl}_{2} \cdot 4 \mathrm{H}_{2} \mathrm{O}$, respectivamente (Alvarez V., 1974).

$\mathrm{Na}$ segunda etapa do experimento, oito meses após o plantio, o anel inferior dos tratamentos FB, FM e FA foi retirado, por meio de um corte transversal na coluna, levando à poda do sistema radicular, e substituído por outro anel com a mesma amostra de solo LVA e, que para o tratamento FM2, apenas se adicionou o anel inferior (terceiro anel). Nestes anéis inferiores, foram aplicadas quatro adubações localizadas, distribuídas uniformemente em todo o volume de solo: adubação baixa (AB), adubação média (AM), adubação alta (AA) e adubação média mais esterco de galinha (AM2) (Quadro 4), correspondentes às recomendações para o terceiro ano após o plantio (Freire et al., 1984).

Da quantidade total de cloreto de potássio e uréia recomendada, apenas $40 \%$ foi adicionada ao solo do anel inferior, buscando simular a adubação em sulcos. Os $60 \%$ restantes foram parcelados em duas aplicações em cobertura.

Cerca de 90 dias após a colocação do anel inferior, realizou-se uma adubação foliar com oxicloreto de cobre $3 \mathrm{~g} \mathrm{~L}^{-1}$, após serem observados sintomas de deficiência desse elemento.

Quadro 3. Adubação básica de plantio adicionada ao solo do anel superior para os diferentes níveis de fertilidade ${ }^{(1)}$

\begin{tabular}{|c|c|c|c|c|c|}
\hline \multirow{2}{*}{ Nível de fertilidade ${ }^{(2)}$} & \multirow{2}{*}{$\frac{\text { Superf. triplo }}{\text { Plantio }}$} & \multicolumn{2}{|c|}{ Uréia } & \multicolumn{2}{|c|}{$\mathrm{KCl}$} \\
\hline & & Plantio & Cobertura & Plantio & Cobertura \\
\hline & $\mathrm{mg} \mathrm{dm}^{-3}$ de $\mathrm{P}$ & \multicolumn{2}{|c|}{$\longrightarrow \mathrm{mg} \mathrm{dm}^{-3}$ de $\mathrm{N}$} & \multicolumn{2}{|c|}{$\mathrm{mg} \mathrm{dm}^{-3}$ de $\mathrm{K}$} \\
\hline FB & 612 & 64 & 191 & 97,5 & 292,5 \\
\hline FM & 408 & 64 & 191 & 65,0 & 195,0 \\
\hline FM2 & 408 & 64 & 191 & 65,0 & 195,0 \\
\hline FA & 204 & 64 & 191 & 32,5 & 97,5 \\
\hline
\end{tabular}

(1) Adubação equivalente à recomendação de Freire et al. (1984), considerando-se o volume da cova padrão (64 $\left.\mathrm{dm}^{3}\right)$ e o volume do anel $\left(4,71 \mathrm{dm}^{3}\right) .{ }^{(2)} \mathrm{FB}=$ fertilidade baixa; FM= fertilidade média; FM2= fertilidade média, sem corte de raízes; FA= fertilidade alta.

Quadro 4. Quantidades de adubos aplicadas no solo do anel inferior e em cobertura correspondentes às diferentes adubações localizadas ${ }^{(1)}$

\begin{tabular}{|c|c|c|c|c|c|c|c|}
\hline \multirow{2}{*}{$\begin{array}{l}\text { Nível de adubą ão } \\
\text { loc alieada }\end{array}$} & \multirow{2}{*}{$\begin{array}{l}\text { Esterco } \\
\text { galinha }\end{array}$} & \multirow{2}{*}{$\begin{array}{l}\text { Mistura } \\
\text { corretiva }\end{array}$} & \multirow{2}{*}{$\begin{array}{l}\text { Superfosf ato } \\
\text { Triplo }\end{array}$} & \multicolumn{2}{|c|}{ Uréia ${ }^{(8)}$} & \multicolumn{2}{|c|}{$\mathrm{KCl}^{(3)}$} \\
\hline & & & & $\mathrm{AI}$ & $\mathrm{CB}$ & $\mathrm{AI}$ & $\mathrm{CB}$ \\
\hline & \multicolumn{2}{|c|}{$\longrightarrow \mathrm{tha}^{\mathrm{l}}$} & $\mathrm{mgdm} \cdot 8$ de $\mathrm{P}$ & \multicolumn{2}{|c|}{$-\mathrm{m} g \mathrm{dm} \cdot s \mathrm{de} \mathrm{N}_{-}$} & \multicolumn{2}{|c|}{$-m g d m^{s}$ de $K-$} \\
\hline$A B$ & 0 & 1,18 & 20,5 & 125 & 187,5 & 62 & 98 \\
\hline$A M$ & 0 & 8,55 & 41,0 & 125 & 187,5 & 104 & 156 \\
\hline AM2 & 10 & 8,55 & $\$ 1,0$ & 125 & 187,5 & 104 & 156 \\
\hline$A A$ & 0 & 5,91 & 61,5 & 125 & 187,5 & 145 & 218 \\
\hline
\end{tabular}

(1) Adubação equivalente à recomendação de Freire et al. (1984), para o $3^{\circ}$ ano de plantio, considerando-se o volume da coluna $\left(14,13 \mathrm{dm}^{3}\right)$ e da cova $\left(320 \mathrm{dm}^{3}\right) .{ }^{(2)} \mathrm{AB}=$ Adubação baixa; $\mathrm{AM}=$ Adubação média; AM2 = Adubação média mais esterco de galinha; $\mathrm{AA}=$ Adubação alta. ${ }^{(3)} \mathrm{AI}=$ Anel inferior; $\mathrm{CB}=$ Em cobertura. 
Dezessete meses após o plantio, foram feitas avaliações do diâmetro da copa e do caule, da altura da planta e do número de ramos, para, em seguida, efetuar o corte da parte aérea.

Os anéis de cada coluna foram desmontados, separando-se as raízes do solo e classificando-as em finas (<1,0 mm de diâmetro) e grossas, conforme avaliação visual.

O material vegetal foi seco em estufa com circulação forçada de ar a $70^{\circ} \mathrm{C}$, até peso constante, determinando-se o peso da matéria seca.

Nas análises de variância para altura de plantas, número de ramos e produções de matéria seca da parte aérea e de raízes finas e grossas dos diferentes anéis, os efeitos médios dos níveis de fertilidade e os efeitos simples das adubações dentro dos níveis de fertilidade foram desdobrados em graus individuais de liberdade.

\section{RESULTADOS E DISCUSSÃO}

\section{Efeito dos níveis de fertilidade}

No quadro 5, verifica-se o efeito de níveis de fertilidade (representados pelos níveis de calagem e doses de esterco de galinha aplicados para incubação das amostras de solo e da adubação de plantio) e da adubação localizada no anel de substituição sobre o desenvolvimento da parte aérea de plantas de cafeeiro cultivar Catuaí Vermelho.
A altura das plantas e o número de ramos decresceram linearmente com o aumento de nível de fertilidade gerado. Este efeito deveu-se, essencialmente, à adubação de plantio adicionada ao anel superior, em razão de ter sido maior para o nível de fertilidade baixo e menor para o nível alto, especialmente para o P (Quadro 3). Para a matéria seca da parte aérea, esta tendência também foi observada. Entretanto, este efeito não atingiu a significância, possivelmente em decorrência dos elevados valores de coeficiente de variação obtidos (Quadro 6). O crescimento do sistema radicular das plantas, por sua vez, em média, não foi afetado pelos níveis de fertilidade (Quadro 7).

Os resultados evidenciam que, para o crescimento da parte aérea, as adubações de plantio para os níveis de fertilidade baixo (FB) e médio (FM) foram adequadas e suficientes para elevar a disponibilidade de nutrientes a um nível satisfatório. Entretanto, para o nível de fertilidade alto (FA), parece ter havido algum fator limitante, mesmo após a adubação de plantio, mostrando que, na geração deste nível de fertilidade, a disponibilidade de nutrientes não foi satisfatória, ou que a dose de adubação adicionada foi aquém da necessária. É possível que o nutriente limitante tenha sido o $\mathrm{P}$, sendo a adubação fosfatada de plantio insuficiente para estabelecer um bom equilíbrio com os demais nutrientes (Alvarez V. et al., 1987), visto que, na fase inicial, pretendia-se obter plantas com bom crescimento para a fase de poda de raíz.

Quadro 5. Altura das plantas, número de ramos e produção de matéria seca da parte aérea de cafeeiros, considerando os níveis de fertilidade e da adubação localizada

\begin{tabular}{|c|c|c|c|c|}
\hline \multirow{2}{*}{ Nível da adubação localizada(2) } & \multicolumn{4}{|c|}{ Nível de fertilidade $e^{(1)}$} \\
\hline & FB & FM & FM2 & FA \\
\hline & \multicolumn{4}{|c|}{ - Altura das plantas, cm } \\
\hline $\mathrm{AB}$ & 79,1 & 87,4 & 83,3 & 58,8 \\
\hline $\mathrm{AM}$ & 74,5 & 83,9 & 87,8 & 64,5 \\
\hline $\mathrm{AM} 2$ & 81,6 & 63,3 & 82,0 & 59,8 \\
\hline $\mathrm{AA}$ & 81,1 & 78,3 & 78,0 & 90,2 \\
\hline \multirow[t]{2}{*}{ Média } & 79,1 & 78,2 & 82,8 & 68,3 \\
\hline & \multicolumn{4}{|c|}{ Número de ramos } \\
\hline $\mathrm{AB}$ & 25,0 & 21,8 & 21,5 & 14,3 \\
\hline $\mathrm{AM}$ & 21,5 & 22,3 & 23,3 & 17,8 \\
\hline $\mathrm{AM} 2$ & 22,8 & 16,3 & 22,5 & 16,8 \\
\hline $\mathrm{AA}$ & 21,5 & 21,3 & 19,8 & 24,3 \\
\hline \multirow[t]{2}{*}{ Média } & 22,7 & 20,4 & 21,8 & 18,3 \\
\hline & \multicolumn{4}{|c|}{ - Matéria seca da parte aérea, g planta ${ }^{-1}$} \\
\hline $\mathrm{AB}$ & 68,0 & 73,9 & 73,4 & 47,9 \\
\hline $\mathrm{AM}$ & 72,7 & 100,6 & 107,3 & 51,0 \\
\hline $\mathrm{AM} 2$ & 84,8 & 52,2 & 96,0 & 46,7 \\
\hline $\mathrm{AA}$ & 77,3 & 68,2 & 74,3 & 103,6 \\
\hline Média & 75,7 & 73,8 & 87,7 & 62,3 \\
\hline
\end{tabular}

(1) $\mathrm{FB}=$ fertilidade baixa; FM = fertilidade média; FM2 = fertilidade média, sem corte de raízes; $\mathrm{FA}=$ fertilidade alta. ${ }^{(2)}$ AB = adubação baixa; AM = adubação média; AM2 = adubação média mais esterco de galinha; AA= adubação alta. 
Quadro 6. Análise de variância para altura das plantas (cm), número de ramos e produção de matéria seca da parte aérea das plantas de cafeeiro $\left(\mathrm{g}_{\text {planta }}{ }^{-1}\right)$

\begin{tabular}{|c|c|c|c|c|}
\hline \multirow{2}{*}{ Fonte de variação( ${ }^{(1)}$} & \multirow{2}{*}{ GL } & \multicolumn{3}{|c|}{ Quadrado médio } \\
\hline & & Altura de planta & Número de ramos & Matéria seca da parte aérea \\
\hline Blocos & 3 & $940,02^{*}$ & 65,68 & $3.208,40$ \\
\hline $\mathrm{NF} 1$ & 1 & $935,28 *$ & $157,53^{*}$ & $1.434,87$ \\
\hline $\mathrm{NF}_{q}$ & 1 & 228,17 & 0,09 & 238,58 \\
\hline Corte raíz d/FM & 1 & 124,82 & 15,13 & $1.562,69$ \\
\hline $\mathrm{A}_{1} \mathrm{~d} / \mathrm{FB}$ & 1 & 8,00 & 24,50 & 172,14 \\
\hline $\mathrm{A}_{q} \mathrm{~d} / \mathrm{FB}$ & 1 & 84,38 & 8,17 & 0,02 \\
\hline M.O. d/AM d/FB & 1 & 101,53 & 3,13 & 291,97 \\
\hline $\mathrm{A}_{1} \mathrm{~d} / \mathrm{FM}$ & 1 & 148,78 & 0,50 & 65,95 \\
\hline $\mathrm{A}_{\mathrm{q}} \mathrm{d} / \mathrm{FM}$ & 1 & 1,76 & 1,50 & $2.333,47$ \\
\hline M.O. d/AM d/FM & 1 & $850,78 *$ & $72,00^{\circ}$ & $4.688,99^{\circ}$ \\
\hline $\mathrm{A}_{1} \mathrm{~d} / \mathrm{FM} 2$ & 1 & 56,18 & 6,13 & 1,59 \\
\hline $\mathrm{A}_{\mathrm{q}} \mathrm{d} / \mathrm{FM} 2$ & 1 & 69,36 & 18,38 & $2.991,10$ \\
\hline M.O. d/AM d/FM 2 & 1 & 28,13 & 1,13 & 256,06 \\
\hline $\mathrm{A}_{1} \mathrm{~d} / \mathrm{FA}$ & 1 & $1.968,78^{* * *}$ & $200,00 * *$ & $6.195,52 *$ \\
\hline $\mathrm{A}_{q} \mathrm{~d} / \mathrm{FA}$ & 1 & 263,34 & 6,00 & $1.632,68$ \\
\hline M.O. d/AM d/FA & 1 & 45,13 & 2,00 & 36,51 \\
\hline Erro & 45 & 207,46 & 23,67 & $1.341,31$ \\
\hline C.V.(\%) & & 18,71 & 23,43 & 48,91 \\
\hline
\end{tabular}

${ }^{(1)} \mathrm{NF}=$ Nível de fertilidade: FB = Fertilidade baixa; FM = Fertilidade média; FM2 = Fertilidade média, sem corte de raízes; FA = Fertilidade alta. A = Adubação; $\mathrm{AM}=$ Adubação média localizada; M.O. = Matéria orgânica (esterco de galinha); ${ }_{1}=$ linear; $_{q}=$ quadrático; $\mathrm{d} /=$ dentro de; ${ }^{\circ},{ }^{*},{ }^{* *}, * * *=$ significativos a 10,$0 ; 5,0 ; 1,0$ e $0,1 \%$.

\section{Efeito do corte de raízes}

O efeito do corte de raízes pode ser observado, comparando os resultados obtidos com os tratamentos que compõem os níveis de fertilidade FM e FM2.

Das características avaliadas na parte aérea, a altura das plantas e o número de ramos não foram influenciados pelo corte de raízes (Quadros 5 e 6). Entretanto, a produção de matéria seca mostrou, em média, tendência de redução de acordo com o corte, decorrente do efeito negativo quando da adição de matéria orgânica (AM2).

Durante a colheita do experimento, por meio de observações visuais do sistema radicular, verificouse que o corte de raízes não prejudicou o seu desenvolvimento, uma vez que se constatou uma eficiente rebrota e crescimento a partir do ponto de corte (Figura 1).

Esse resultado traz implicações diretas com as práticas agrícolas adotadas na condução da cultura. De acordo com Franco \& Inforzato (1946), os cultivos profundos podem causar cortes de radicelas e os danos serão tanto maiores quanto mais superficial for o sistema radicular. Os resultados obtidos indicam que o corte das raízes decorrente da abertura de um sulco para as adubações fosfatadas anuais não resulta em seqüelas para as plantas, uma vez que a rebrota de raízes é intensa. Por outro lado, Rena \& Maestri (1986) argumentam que, no caso de a injúria ao sistema radicular ser repetida anualmente, pode ocorrer um completo debilitamento da planta. Nesse sentido, novas pesquisas em condições de campo poderiam demonstrar, com mais exatidão, a ocorrência ou não deste debilitamento.

Por outro lado, analisando o efeito do corte de raízes dentro da adubação localizada, verificou-se que, onde a matéria orgânica foi adicionada, o corte reduziu a produção de matéria seca de raízes nos diferentes anéis (Quadros 7 e 8). Conseqüentemente, a mesma tendência pôde ser observada para o crescimento da parte aérea, em especial, a produção de matéria seca (Quadros 5 e 6). Cannell (1971), ao estudar o crescimento do sistema radicular de cafeeiros no Quênia, verificou maior taxa de crescimento radicular em períodos quentes e secos do ano e, com o início da estação chuvosa, as raízes principalmente as de diâmetro superior a $3 \mathrm{~mm}$, praticamente paralisavam seu crescimento. Maior crescimento do sistema radicular em certos períodos, quando o conteúdo de umidade do solo não era tão alto, também foi verificado por Beckley (1935). Este fato evidencia que as adubações no sulco, com adição de resíduos orgânicos, devem realizar-se na época seca, pouco antes do início das chuvas, quando o crescimento do sistema radicular é intenso.

Verificou-se também uma interação negativa entre adubação orgânica e corte de raízes, quando se estudou o efeito da matéria orgânica dentro de adubação localizada para o nível de fertilidade médio (FM). Esse efeito foi significativo para todas as características da parte aérea e, embora a produção de matéria seca das raízes apresentasse a mesma 
Quadro 7. Produção de matéria seca de raízes finas e grossas, nos diferentes anéis, considerando a fertilidade e a adubação localizada

\begin{tabular}{|c|c|c|c|c|}
\hline \multirow{2}{*}{ 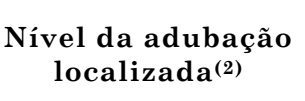 } & \multicolumn{4}{|c|}{ Nível de fertilidade(1) } \\
\hline & FB & FM & FM2 & FA \\
\hline \multicolumn{5}{|c|}{ Raízes finas no anel superior, $\mathrm{g}$} \\
\hline $\mathrm{AB}$ & 0,96 & 1,20 & 0,81 & 0,95 \\
\hline $\mathrm{AM}$ & 1,51 & 2,44 & 2,97 & 1,13 \\
\hline AM2 & 2,05 & 1,33 & 2,69 & 1,05 \\
\hline $\mathrm{AA}$ & 1,34 & 1,32 & 1,85 & 2,63 \\
\hline Média & 1,47 & 1,57 & 2,08 & 1,44 \\
\hline \multicolumn{5}{|c|}{ Raízes grossas no anel superior, g } \\
\hline $\mathrm{AB}$ & 1,26 & 1,31 & 1,39 & 1,38 \\
\hline $\mathrm{AM}$ & 1,81 & 2,53 & 2,53 & 1,58 \\
\hline AM2 & 2,75 & 1,22 & 2,50 & 1,15 \\
\hline AA & 2,16 & 1,68 & 1,89 & 3,14 \\
\hline Média & 2,00 & 1,69 & 2,08 & 1,81 \\
\hline \multicolumn{5}{|c|}{ Raízes finas no anel médio, g } \\
\hline $\mathrm{AB}$ & 0,85 & 0,70 & 1,02 & 0,68 \\
\hline $\mathrm{AM}$ & 1,33 & 1,78 & 2,46 & 0,83 \\
\hline AM2 & 1,67 & 0,93 & 2,53 & 0,75 \\
\hline AA & 1,28 & 1,11 & 1,63 & 2,14 \\
\hline Média & 1,28 & 1,13 & 1,91 & 1,10 \\
\hline \multicolumn{5}{|c|}{ Raízes grossas no anel médio, g } \\
\hline $\mathrm{AB}$ & 1,48 & 1,75 & 1,72 & 1,38 \\
\hline $\mathrm{AM}$ & 2,41 & 2,74 & 3,61 & 1,86 \\
\hline AM2 & 2,76 & 1,40 & 3,31 & 1,31 \\
\hline AA & 2,34 & 1,86 & 2,04 & 2,83 \\
\hline Média & 2,25 & 1,94 & 2,67 & 1,85 \\
\hline \multicolumn{5}{|c|}{ Raízes finas no anel inferior, g } \\
\hline $\mathrm{AB}$ & 1,77 & 1,98 & 1,86 & 0,93 \\
\hline $\mathrm{AM}$ & 1,15 & 2,54 & 2,83 & 0,72 \\
\hline AM2 & 1,98 & 0,99 & 2,28 & 0,57 \\
\hline $\mathrm{AA}$ & 1,16 & 1,10 & 1,28 & 1,77 \\
\hline Média & 1,52 & 1,65 & 2,06 & 1,00 \\
\hline \multicolumn{5}{|c|}{ Raízes grossas no anel inferior, g } \\
\hline $\mathrm{AB}$ & 1,51 & 2,08 & 1,87 & 0,87 \\
\hline $\mathrm{AM}$ & 1,86 & 3,38 & 3,51 & 0,99 \\
\hline AM2 & 2,22 & 0,96 & 3,57 & 0,93 \\
\hline AA & 1,49 & 1,39 & 1,57 & 2,10 \\
\hline Média & 1,77 & 1,95 & 2,63 & 1,22 \\
\hline
\end{tabular}

(1) FB = Fertilidade baixa; FM = Fertilidade média; FM2 = Fertilidade média sem corte de raízes; FA = Fertilidade alta. ${ }^{(2)} \mathrm{AB}=$ Adubação baixa; $\mathrm{AM}=$ Adubação média; $\mathrm{AM} 2$ = Adubação média mais esterco de galinha; $\mathrm{AA}=$ Adubação alta.

tendência, não se obteve significância, talvez em razão dos elevados coeficientes de variação (dados não apresentados). Esse efeito apenas no nível de fertilidade médio (FM), quando a produção tendeu a ser maior, revela que, nos níveis de fertilidade FB e FA, outro fator deve ter limitado a produção.

\section{Efeito da adubação localizada}

Para o nível de fertilidade baixo (FB), não foi observado efeito significativo de adubação, nem sobre o crescimento da parte aérea (Quadros 5 e 6), nem sobre o crescimento das raízes finas ou grossas (Quadros 7 e 8). Esses resultados evidenciam que a adubação de plantio baseada no nível de fertilidade gerado foi adequada, já que a produção não aumentou com o aumento das doses de adubação localizada.

Entretanto, para o nível de fertilidade alto (FA), este comportamento não foi observado, uma vez que o crescimento da parte aérea aumentou linearmente com o incremento das doses de adubação localizada, notadamente para a matéria seca produzida (Quadro 5), sendo esta tendência igualmente verificada para a produção de matéria seca de raízes (Quadro 8). O menor crescimento do sistema radicular do cafeeiro em solos de baixa fertilidade foi primeiramente observado há cerca de 70 anos por Nutman (1933).

No nível de fertilidade médio (FM), observou-se que para a produção de matéria seca da parte aérea houve uma tendência de resposta quadrática positiva à aplicação da adubação localizada, não tendo este efeito atingido a significância, possivelmente por causa do elevado valor do coeficiente de variação. Para a produção de matéria seca de raízes (Quadro 7), observou-se a mesma tendência de resposta quadrática.

A tendência de efeito quadrático da adubação localizada, observada dentro do nível de fertilidade médio (FM), mostrou ter sido a adubação de plantio mais equilibrada com o nível de fertilidade gerado inicialmente, uma vez que a adubação média utilizada no anel inferior proporcionou a melhor produção dentro de FM.

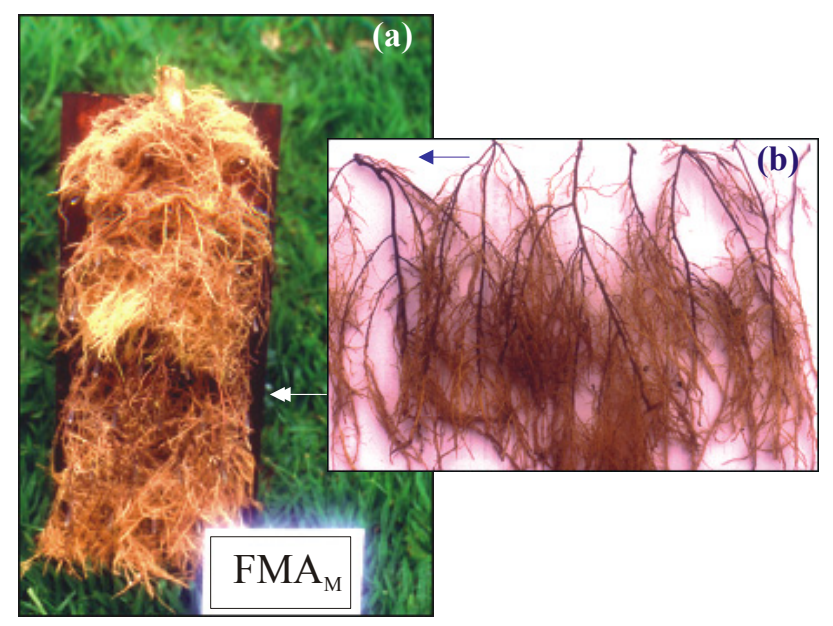

Figura 1. Crescimento do sistema radicular nas colunas de solo, destacando-se a população de raízes no terceiro anel (a) e rebrota das raízes após a realização da poda (b). 
Quadro 8. Análise de variância para produção de matéria seca de raízes finas (RF) e grossas (RG) de plantas de cafeeiro (g) nos anéis: superior (S), médio (M) e inferior (I) das colunas de solo

\begin{tabular}{|c|c|c|c|c|c|c|c|}
\hline \multirow{2}{*}{ Fonte de variação } & \multirow{2}{*}{ GL } & \multicolumn{6}{|c|}{ Quadrado médio } \\
\hline & & RFS & RGS & RFM & RGM & RFI & RGI \\
\hline Blocos & 3 & 2,0306 & 1,6018 & 1,8159 & $5,0997 *$ & 2,0510 & $6,7286^{*}$ \\
\hline $\mathrm{NF}_{1}$ & 1 & 0,0072 & 0,2888 & 0,2592 & 1,2800 & 2,1632 & 2,4200 \\
\hline $\mathrm{NF}_{q}$ & 1 & 0,1411 & 0,4931 & 0,0384 & 0,1291 & 1,6224 & 2,2083 \\
\hline Corte raíz d/FM & 1 & 2,0808 & 1,2168 & $4,8672^{*}$ & $4,2632^{*}$ & 1,3448 & $3,6992^{0}$ \\
\hline $\mathrm{A} l \mathrm{~d} / \mathrm{FB}$ & 1 & 0,2888 & 1,6200 & 0,3698 & 1,4792 & 0,7442 & 0,0008 \\
\hline $\mathrm{A}_{\mathrm{q}} \mathrm{d} / \mathrm{FB}$ & 1 & 0,3456 & 0,0267 & 0,1873 & 0,6667 & 0,2646 & 0,3456 \\
\hline M.O. d/AM d/FB & 1 & 0,5632 & 1,7672 & 0,2312 & 0,2450 & 1,3778 & 0,2592 \\
\hline $\mathrm{A}_{1} \mathrm{~d} / \mathrm{FM}$ & 1 & 0,0288 & 0,2738 & 0,3362 & 0,0241 & 1,5488 & 0,9522 \\
\hline $\mathrm{A}_{\mathrm{q}} \mathrm{d} / \mathrm{FM}$ & 1 & $3,7131^{0}$ & $2,8586^{0}$ & 2,0417 & 2,3313 & 2,6667 & $7,2161^{*}$ \\
\hline M.O. d/AM d/FM & 1 & 2,4642 & $3,3222^{0}$ & 1,4450 & $3,5912^{0}$ & $4,8050^{*}$ & $11,7128^{* *}$ \\
\hline $\mathrm{A}_{1} \mathrm{~d} / \mathrm{FM} 2$ & 1 & 2,1632 & 0,5000 & 0,7442 & 0,2048 & 0,6728 & 0,1800 \\
\hline $\mathrm{A}_{\mathrm{q}} \mathrm{d} / \mathrm{FM} 2$ & 1 & $7,1723^{*}$ & 2,1123 & $3,4353^{0}$ & $7,9811^{* *}$ & $4,2336^{*}$ & $8,5443^{* *}$ \\
\hline M.O. d/AM d/FM2 & 1 & 0,1568 & 0,0018 & 0,0098 & 0,1800 & 0,6050 & 0,0072 \\
\hline $\mathrm{A}_{1} \mathrm{~d} / \mathrm{FA}$ & 1 & $5,6448^{*}$ & $6,1952^{*}$ & $4,2632^{*}$ & $4,2050^{*}$ & 1,4112 & $3,0258^{0}$ \\
\hline $\mathrm{A}_{\mathrm{q}} \mathrm{d} / \mathrm{FA}$ & 1 & 1,1616 & 1,2331 & 0,8971 & 0,1601 & 1,0584 & 0,6534 \\
\hline M.O. d/AM d/FA & 1 & 0,0128 & 0,3698 & 0,0128 & 0,6050 & 0,0450 & 0,0072 \\
\hline Erro & 45 & 1,7647 & 1,5719 & 1,0278 & 1,6439 & 1,7446 & 2,8955 \\
\hline C.V.(\%) & & 81,203 & 66,186 & 74,827 & 59,060 & 84,924 & 89,861 \\
\hline
\end{tabular}

NF = Nível de fertilidade: $\mathrm{FB}=$ Fertilidade baixa; $\mathrm{FM}=$ Fertilidade média; FM2 = Fertilidade média, sem corte de raízes; FA = Fertilidade alta. A = Adubação; $\mathrm{AM}=$ Adubação média localizada; M.O. = Matéria orgânica (esterco de galinha); ${ }_{1}=$ linear; $_{q}=$ quadrático; $\mathrm{d} /=$ dentro de; ${ }^{\circ},{ }^{*}, * *=$ significativos a 10,$0 ; 5,0$ e $1,0 \%$.

No nível de fertilidade médio sem corte de raízes (FM2), observou-se também tendência de resposta quadrática à adubação localizada, onde o efeito foi significativo para raízes finas no anel superior e para raízes finas e grossas no anel médio. Esses resultados demonstram que, no nível de fertilidade médio, o fato de não se fazer o corte de raízes acentuou o efeito quadrático da adubação localizada.

\section{CONCLUSÕES}

1. A altura das plantas e o número de ramos decresceram linearmente com o aumento do nível de fertilidade gerado, em razão das menores doses de $\mathrm{P}$ e $\mathrm{K}$ adicionadas na adubação de plantio. Esta tendência também foi verificada para produção de matéria seca da parte aérea.

2. O crescimento do sistema radicular não foi afetado pelos níveis de fertilidade.

3. O crescimento e a produção de matéria seca da parte aérea e das raízes não foram influenciados pela poda das raízes, exceto quando se adicionou esterco de galinha na adubação localizada no nível de fertilidade médio, quando se observou efeito negativo.

4. As adubações localizadas no nível de fertilidade baixo não influíram no crescimento da parte aérea nem do sistema radicular; no nível médio, verificou- se efeito quadrático positivo da adubação localizada e, no nível de fertilidade alto, observou-se aumento linear com o incremento da adubação localizada no crescimento das plantas de cafeeiro cultivar Catuaí Vermelho, mostrando que as adubações de plantio baseadas nos níveis de fertilidade gerados foram adequadas no nível baixo, equilibradas no nível médio e insuficientes no nível alto.

\section{LITERATURA CITADA}

ALVAREZ V., V.H. Equilíbrio de formas disponíveis de fósforo e enxofre em dois Latossolos de Minas Gerais. Viçosa, Universidade Federal de Viçosa, 1974. 125p. (Tese de Mestrado)

ALVAREZ V., V.H.; FREIRE, F.M. \& GUIMARÃES, P.T.G. Concentrações relativas ótimas de nitrogênio, fósforo e enxofre, na adubação do cafeeiro, num Latossolo VermelhoEscuro de Machado, MG. Pesq. Agropec. Bras., 22:145-152, 1987.

BAR-TAL, A.; FEIGIN, A.; SHEINFELD, S.; ROSENBERG, R.; STERNBAUM, B.; RYLSKI, I. \& PRESSMAN, E. Root restriction and $\mathrm{N}-\mathrm{NO}_{3}$ solution concentration effects on nutrient uptake, transpiration and dry matter production of tomato. Sci. Hortic., 63:195-208. 1995.

BECKLEY, V.A. Observations on coffee in Kenya. I. Chlorosis and die-back in coffee. Emp. J. Expl. Agric., 3:203-209, 1935.

CANNELL, M.G.R. Production of dry matter in trees of Coffea arabica L. in Kenya as affected by seasonal climatic differences and the presence of fruits. Ann. Appl. Biol., 67:99-120, 1971. 
DA MATTA, F.M. \& AMARAL, J.A.T. \& RENA, A.B. Growth periodicity in trees of Coffea arabica L. in relation to nitrogen supply and nitrate reductase activity. Field Crops Res., 60:223-229, 1999.

DEMATTÊ, J.L.I. Characteristics of brazilian soils related to root growth. In: RUSSEL, R.S.; IGUE, K. \& METHA, Y.R., eds. The soil root system in relation to brazilian agriculture. Londrina, IAPAR, 1981. p.21-41.

FERNANDES, B. Retenção e movimento de água no solo. Viçosa, Universidade Federal de Viçosa., 1967. 48p. (Tese de Mestrado)

FONSECA, D.M. Níveis críticos de fósforo para o estabelecimento de Andropogon gayanus var. bisquamulatus, Panicum maximum e Brachiaria decumbens, em casa de vegetação e no campo. Viçosa, Universidade Federal de Viçosa, 1995. 150p. (Tese de Doutorado)

FRANCO, C.M. \& INFORZATO, R. O sistema radicular do cafeeiro nos principais tipos de solo do Estado de São Paulo. Bragantia, 6:443-478, 1946.

FREIRE, F.M.; GUIMARÃES, P.T.G.; ALVAREZ V., V.H. \& MELLES, C.A. Calagem, gessagem e adubação do cafeeiro. Belo Horizonte, EPAMIG, 1984. 20p. (Boletim Técnico, 11)

JACKSON, M.L. Análisis químico de suelos. 3.ed. Barcelona, Omega, 1976. 662p.

LEITE, P.B. Níveis críticos de fósforo para milho e eucalipto, em função da sua localização no solo, em vasos. Viçosa, Universidade Federal de Viçosa., 1991. 107p. (Tese de Mestrado)

LOPES, A.S. Solos sob "cerrado": Características, propriedades e manejo. Piracicaba, Instituto da Potassa, 1983. 162p.
NUTMAN, F.J. The root system of Coffea arabica L. II. The effect of some soil conditions in modifying the "normal" rootsystem. Emp. J. Expl. Agric., 1:285-296, 1933.

ONG, C.K.; WILSON, J.; DEANS, J.D.; MULAYTA, J.; RAUSSEN, T. \& WAJJA-MUSUKWE, N. Tree-crop interaction: manipulation of water use and root function. Agric. Waste Manag., 53:171-186, 2002.

POZZA, A.A.A.; GUIMARÃES, P.T.G.; ROMANIELLO, M.M.; POZZA, E.A. \& CARVALHO, J.G. Suprimento de fósforo na produção e intensidade da cercosporiose de mudas de cafeeiro em tubetes. Ci. Agrotec., 26:5:970-976, 2002.

RENA, A.B. \& MAESTRI, M. Fisiologia do cafeeiro. In: RENA, A.B.; MALAVOLTA, E.; ROCHA, M. \& YAMADA, T.Y., eds. Cultura do cafeeiro: Fatores que afetam a produtividade. Piracicaba, Assiação Brasileira de Pesquisa de Potássio e Fósforo, 1986. p.13-85.

RITCHEY, K.D.; SOUSA, D.M.G.; LOBATO, E. \& CORREA, C. Calcium leaching to increase rooting depth in Brazilian Savannah Oxisol. Agron. J., 72:40-44,1980.

SILVA, J.V. Contribution for the study of root system development in some soils in São Tomé. Est. Agron., 1:225$257,1960$.

SUAREZ DE CASTRO, F. \& RODRIGUEZ, G.A. Distribución de las raíces de los cafetos. Agricultura de las Américas, 5:42-46, 1963.

VETTORI, L. Métodos de análise de solo. Rio de Janeiro, Ministério da Agricultura, 1969. 24p.(Boletim Técnico, 7)

WOODALL, G.S. \& WARD, B.H. Soil water relations, crop production and root pruning of a belt of trees. Agric. Waste Manag., 53:153-169, 2002. 